\title{
Protée
}

\section{La mort de Müllière ou à quoi ressemble un chant du cygne}

\section{Dror Harari}

Volume 27, numéro 1, 1999

La Mort de Molière et des autres

URI : https://id.erudit.org/iderudit/030549ar

DOI : https://doi.org/10.7202/030549ar

Aller au sommaire du numéro

Éditeur(s)

Département des arts et lettres - Université du Québec à Chicoutimi

ISSN

0300-3523 (imprimé)

1708-2307 (numérique)

Découvrir la revue

Citer cet article

Harari, D. (1999). La mort de Müllière ou à quoi ressemble un chant du cygne. Protée, 27(1), 99-104. https://doi.org/10.7202/030549ar

\section{Résumé de l'article}

Cet article identifie les diverses traces laissées par une présence masquée, qui se sert de l'image de Molière mourant comme d'un symptôme déplacé de sa propre mort anticipée. Quelqu'un manipule les signes de la biographie de Molière et de sa création artistique de façon à parler de lui-même. d'utilisation que vous pouvez consulter en ligne.

https://apropos.erudit.org/fr/usagers/politique-dutilisation/ 


\section{LA MORT DE MÜLLIÈRE OU À QUOI RESSEMBLE UN CHANT DU CYGNE \\ Traduit de I'anglais par Jean-Pierre Vidal}

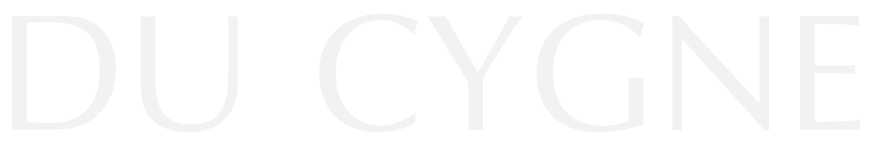

DROR HARARI

Dans le texte qui suit, je me propose de soumettre au lecteur quelques idées troublantes qui m'ont hanté pendant tout le visionnement de La Mort de Molière ${ }^{1}$, dernière œuvre produite en collaboration par le duo Wilson-Müller. Je crois important de souligner dès maintenant que ces incertitudes (qui n'ont pas encore atteint le confortable statut d'hypothèses) représentent ma réaction à quelques-uns seulement des «nœuds " ${ }^{2}$ qui tissent le véritable patchwork qu'est cette œuvre. Je n'ai nullement l'intention de proposer une solution d'ensemble à ce qui se présente comme une énigme soigneusement construite, une mystérieuse manifestation de création hybride, et je ne crois pas non plus qu'une telle entreprise soit souhaitable. Les efforts entrepris pour essayer de convenir d'une stratégie méthodologique avec laquelle affronter cette complexité de contenu et de texture ont fini par me faire prendre conscience que, devant une telle œuvre, la meilleure stratégie consistait peut-être à utiliser autant de tactiques différentes que possible, une option qui fait écho à la vieille croyance sceptique de Nietszche, selon laquelle plus nous serons capables de porter de regards, de regards différents, sur la même chose, plus complet sera notre concept de cette chose (1994). Ici, cependant, je suis davantage poussé par le soupçon que par le scepticisme.

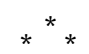

On peut soutenir que les œuvres de Robert Wilson et Heiner Müller forment un champ culturel autosuffisant, bien identifié, cartographié et répertorié.

Comme tel, chacun produit de plein droit ce que Barthes, dans La Chambre claire (1980), appelle un «studium», c'est-à-dire que Wilson, tout comme Müller, suscitent un certain horizon d'attentes basé principalement sur la familiarité que j'ai avec plusieurs de leurs productions, interviews, textes et essais. Ce savoir (filtré par le discours savant) permet de s'orienter (ou de se désorienter) dans cet espace de création.

Pourtant, dans La Mort de Molière, je crois identifier, à côté des voies balisées (et fort fréquentées par d'autres), d'autres traces (laissées inconsciemment) qui témoignent de la présence de ce que Barthes appelle un «punctum»: «[un élément] 
qui part de la scène, comme une flèche, et vient me percer» (ibid., p. 49). Il y a dans cette œuvre quelque chose qui vient troubler l'apparente transparence discursive du «studium» et oblige d'y revenir «voir». Ce «punctum» disruptif fait que l'œuvre agit par les pensées dérangeantes et douloureuses qu'elle suscite. La plus troublante de ces pensées prend la forme d'une série de questions: se peut-il qu'une image artistique de Molière à l'agonie se change en une imago (l'origine étymologique latine du mot «image", avec son sens spécifique de "masque mortuaire») cachant le visage de quelqu'un d'autre? Ou, pour le dire plus crûment, se peut-il que la description quasi biographique de la mort de Molière devienne un bricolage d'images composant l'autobiographie d'un autre mourant (réel, celui-là) qui n'est pas Molière? Et si c'est bien le cas, serait-il faux de prétendre que cette création artistique est soit une élégie, soit une épitaphe? Bien des raisons valables permettraient de mettre un nom sur cette épitaphe, mais je préfère ici tracer les voies performatives de son inscription différée.

Voulezvous que je parle de moi Moi qui

De qui estil question quand

Il est question de moi Qui est ce Moi (Müller, 1990, section 10)

\section{${ }^{*}{ }^{*}$ *}

La Mort de Molière est une œuvre fragmentée, autoreprésentative et à multiples niveaux, dans laquelle sont entre-tissés plusieurs dispositifs autocadrants et divers ordres de représentation. Les mécanismes réflexifs (à la fois textuels et visuels) minent et problématisent de façon signifiante les relations traditionnelles entre le sujet et l'objet (par exemple, Galilée et les étoiles, le médecin et les symptômes, le créateur et sa création), offrant ainsi des lectures complexes qui vont bien au delà de ce qui semble au premier abord le premier motif référentiel de l'œuvre, c'est-à-dire le récit de la mort de Molière.

Dès l'«exposition", Müller (le coauteur) dispose une trappe ontologique: "Ce n'est pas un poème sur
Molière», dit la première ligne du texte. Mais quelques lignes plus bas, cette affirmation s'inverse et l'on nous dit que «c'est un poème sur Molière». La contradiction interne qui marque la séquence d'ouverture vient compliquer encore davantage la lecture d'un passage comme celui-ci:

Le poème observe un mourant au travail

Qui est appellé Molière Le poème n'est pas un film

Le film observe un acteur au travail

Qui représente un homme mourant appellé Molière

(Müller, section 1)

Je soupçonne fort l'exposition de ne pas se borner à indiquer de nombreuses pistes de lectures possibles - tout en soulignant l'impossibilité pour la raison (du moins occidentale) de dénouer complètement ces «nœuds» indécidables -, mais de mettre aussi en place une autoréférence. Bien que Müller affirme sans équivoque qu' «on ne peut pas écrire un poème sur Molière» (ibid.), il nous prouve en fait le contraire. En écrivant un poème sur Molière (réalisant ainsi l'irréalisable), il semble être le seul qui puisse connaître l'issue du labyrinthe ontologique qu'il a luimême créé. Il s'agit là, incidemment, de la première manifestation reconnaissable d'une relation elliptique entre le véritable Auteur de cette pièce et le personnage de Molière (l'Auteur référentiel). Par «relation elliptique», j'entends le fait que cette pièce offre (au moins) deux foyers, dont l'un (Molière) est plus ou moins clairement identifié, alors que l'autre (sur les traces duquel je suis lancé ici) préfère garder l'anonymat. Malheureusement Molière, l'Auteur et le seul qui en principe connaisse la vérité sur sa propre vie et sa création, est en train de mourir. Il semble qu'ainsi l'on nous abandonne dans le labyrinthe, sans carte ni réponses à propos de l'énigme Molière comme de la compréhension de la vidéo qui apparemment porte sur lui. L'Auteur nous donne une leçon (métaphysique) particulièrement dérangeante: «Le résultat / Dépend d'autres circonstances / Que le cours des étoiles». Ainsi la confusion et l'obscurité ne seraient pas un état provisoire auquel succéderait une phase de résolution. Quel soulagement! 
Un autre «nœud» tient au fait qu'ici, à la différence des modes traditionnels de la représentation théâtrale, nous ne sommes pas tout à fait en train de regarder quelqu'un qui représente Molière. Nous sommes plutôt conscients de l'autoreprésentation du Créateur-Wilson jouant Molière. Je pense qu'il s'agit là d'un des buts performatifs-discursifs poursuivis par la section 7 (celle du renard). Alors que presque tous les textes qui composent La Mort de Molière sont des fragments pris à ce qu'on pourrait appeler la littérature canonique (Plutarque, Shakespeare, Kafka, Marlowe, Molière), la section 7 offre une aberration. Elle présente en effet une lettre personnelle écrite par le critique de danse Edwin Denby, dans laquelle celui-ci suggère à son ami Wilson des façons de soigner sa toux. Ce registre consciemment autobiographique, qui s'ouvre de façon tout à fait intime par un "Cher Bob", met en scène et nous rappelle à la fois l'acteur-Créateur qui joue le rôle de Molière. Il sert ainsi de marqueur autoreprésentatif qui, dans une large mesure, mine les effets illusionnistes de l'«histoire" qui nous montre les derniers instants de Molière. À la lumière de cette autoréférentialité, nous voyons le Créateur-acteurWilson incarner l'objet de sa création (le personnage de Molière), dans et avec sa propre image, et ainsi, inévitablement, transcender son apparence au point d'atteindre un lieu situé au delà de la convention ou du faire-semblant artistique. Dans cette sphère où la distinction ténue entre le réel et l'imaginaire se trouve pratiquement effacée, nous pouvons être tentés de reconsidérer le(s) sujet(s) référentiel(s) de cette œuvre: est-ce Molière? Wilson ou Müller? Wilson et Müller? La figure du Créateur?

De plus, dans cette sphère «non représentationnelle» où la coupure ontologique séparant le «je» d'autrui et la vraie vie de l'art devient indistincte, les textes écrits par Müller (le co-Créateur) sont inévitablement suspectés de contenir implicitement des éléments autobiographiques. Et ce soupçon conduit sans doute à de fort troublantes interprétations, puisque «Le poème observe un mourant au travail» (section 1$)$ :
Father, I am dying.
Father, I am dying.
Father, I am dying.
Father, I am dying.
Father, I am dying.
Father, I am dying. (section 5)

S'attacher au pouvoir performatif de l' «exposition" et de la lettre de Denby permet d'indiquer que des mécanismes d'autocadrages sont ici à l'œuvre. Ces mécanismes attirent l'attention sur ce qui semble opérer sous la surface (du faire-semblant, de l'illusion, du signe). J'entr'aperçois le dispositif caché qui l'anime: par exemple les membres de la figure indistincte qui manipule le portrait incliné de Molière, les yeux de l'acteur derrière le masque du renard, le petit enfant qui se cache sous le clavecin. Je deviens même conscient de la main frénétique qui manipule la caméra.

On soupçonne dans une présence absente de se servir de l'identité de Molière, de son image, pour parler d'elle-même. Le personnage de Molière devient le médium vivant d'une présence non présente, invisible et pourtant réelle. Je veux dire par là que si Wilson joue effectivement quelqu'un d'autre que luimême, cet autre référentiel est quelqu'un qui n'est pas Molière.

En termes techniques, je dirai que c'est comme si quelqu'un avait «violé» les lois du signe (stratégie rhétorique que l'on qualifie conventionnellement de «métaphorique» ou «elliptique», cf. Rozik, 1992). Cet acte a en effet perturbé le délicat équilibre qui conventionnellement unit le signe à son référent en permettant aux Créateurs de prendre le contrôle du signe et de le manipuler. Il s'agit là, comme nous l'apprennent certaines mythologies (et l'Art en général), du pouvoir sans partage qu'ont Dieu (les dieux) et le (les) Trickster(s) de déconstruire l'ordre des Choses, de manipuler les signes, en rendant leurs pouvoirs parfaitement incompréhensibles et/ou invisibles. Cette notion est illustrée par une courte séquence où l'on voit des chaises en feu mais qui ne semblent pas se consumer. Cette image fait venir à 
l'esprit l'épisode du «buisson ardent» raconté dans l'Ancien Testament, qui comprend trois des éléments nécessaires à une scène de miracle: le «signe énigmatique", le spectateur confus, et la voix off.

Il pourrait être intéressant à ce stade de faire intervenir un autre «nœud" particulièrement intrigant. Alors que Wilson incarne - ou, pour le dire différemment, signifie avec son propre corps - l'image de quelqu'un d'autre, Müller, lui, (dés)incarne la voix off et l'autorité qu'elle a malgré son enrouement. Compte tenu des mécanismes autoreprésentatifs qui opèrent dans cette œuvre, notre perception de la présence visuelle de Wilson oscille constamment entre une acceptation de l'univers référentiel et sa «dénégation» consciente (cf. Ubersfeld, cité dans Alter, 1990: 63). Wilson n'est pas lui-même (il «est» Molière), mais il n'est pas non plus un autre ${ }^{3}$. On ne peut pas dire, au contraire, que la voix de Müller représente, dans le contexte de l'univers fictionnel (cf. Rozik, 1992) de La Mort de Molière, autre chose qu'ellemême, d'où sa présence. La voix qui, comme le dit Durand, «est inextricablement liée au corps» (1977: 101), rend l'absence du corps de Müller très présente. Elle présente l'irreprésentable.

De mon point de vue, le(s) champ(s) référentiel(s) de «Molière mourant» - qu'on le lise littéralement comme tel, ou de façon allégorique comme une allusion à une autre signification métaphysique (et les deux représentent sans conteste des options raisonnables) -, n'est qu'un leurre. Bien qu'il puisse être un référent en soi ( montrer la mort d'un héros national dans un théâtre national", Linders, 1996: 93), il fonctionne plus comme un symptôme ou comme «le contenu manifeste d'un rêve». Dans ce casci, ajouterai-je, ma position en tant qu'interprète est encore plus problématique que celle de Freud recherchant les mécanismes du «travail du rêve» (pour atteindre l'inatteignable origine inconsciente, son "contenu latent", cf. Freud, 1960: 277-309). Je connais, en effet, tous les détails du «rêve». Je découvre lentement ses mécanismes. Mais le rêveur a choisi de rester non présent, non identifié, non connu. C'est sans doute pourquoi le personnage du médecin moderne est montré perplexe et désorienté quand il examine le fauteuil vide. Après tout, le sujet de son auscultation est absent. Il n'a laissé que les symptômes de sa présence absente.

\section{* *}

Quand je prends en considération tous les «nœuds» mentionnés ci-dessus - la dimension non illusionniste, les mécanismes autoreprésentatifs, le recours à un matériau autobiographique (la lettre de Denby, les propres écrits de Müller), la voix désincarnée et le déconstructeur/manipulateur absent qui se sert de l'image de Molière mourant (son masque mortuaire) pour parler de lui-même -, j'ai peur que l'œuvre ne mette en cause un sujet réel en train de mourir. En un sens, comme le souligne Blau, le performer est toujours en train d'imaginer sa propre mort. Il peut la projeter dans le futur comme un renvoi mais, comme le souvenir, elle semble venir d'en arrière, comme si elle avait déjà eu lieu (1990).

Ce sujet tient ici un rôle performatif et (in)consciemment, peut-être même théâtralement, il prépare, "comme si elle avait déjà eu lieu", sa propre disparition de la scène de ce monde. Comme s'il savait (consciemment ou non) que ce sera là son chant du cygne.

\section{DO YOU REMEMBER DO YOU NO I DONT}

(Müller, section 10) 


\section{Notes}

1. La version de référence ici est celle de la vidéo allemande à laquelle Müller a prêté sa voix (cf. Linders, 1996: 93-95)

2. Il s'agit de ces moments d'intensité dans une représentation qui, si l'on en croit Barba (1990), ne représentent pas nécessairement des moments de résolution mais plutôt attirent l'attention du spectateur et l'incitent à "poser des questions quant à la signification" (p.97).

3. Comme je l'ai déjà indiqué plus haut, Müller attire l'attention sur cette ambiguité dans l'« exposition", à la fois par le fait que «le film observe un acteur au travail» et par l'idée que le poème est écrit sur Molière.

\section{RÉFÉRENCES BIBLIOGRAPHIQUES}

AlTER, J. [1990]: A Sociosemiotic Theory of Theatre, Pennsylvania, University of Pennsylvania Press.

BARBA, E. [1990]: «Four Spectators", The Drama Review, n 1.

BARTHES, R. [1980]: La Chambre claire. Note sur la photographie, Paris, Gallimard/Seuil, "Cahiers du cinéma ".

BlaU, H. [1990] : "Universals of Performance; or Amortizing Play ", dans By Means of Performance: Intercultural Studies of Theatre and Ritual, sous la dir. de W. Appel et R. Schechner, C.U.P.

DuRAND, R. [1977]: «The Disposition of the Voice», dans Performance in Postmodern Culture, sous la dir. de M. Benamou et C. Caramello, Coda Press.

FREUD, S. [1960]: The Interpretation of Dreams, textes traduits et rassemblés par J. Strachey, Basic Books Pub.

LINDERS, J. [1996] : «Molière \pm Müller », Performance Research, vol. 1, $\mathrm{n}^{\circ} 2,93-95$

MÜLleR, H. [1996]: «Texts for The Death of Molière», Performance Research, vol. 1, n² 2, 96-103;

[1990]: «Paysages avec Argonautes», dans Germania Mort à Berlin, Paris, Éd. de Minuit.

NieTszCHE, F. [1994]: On the Genealogy of Morality, sous la dir. de K. Ansell-Pearson, trad. de C. Diethe, C.U.P.

ROZIK, E. [1992]: The Language of Theatre, University of Glasgow. 


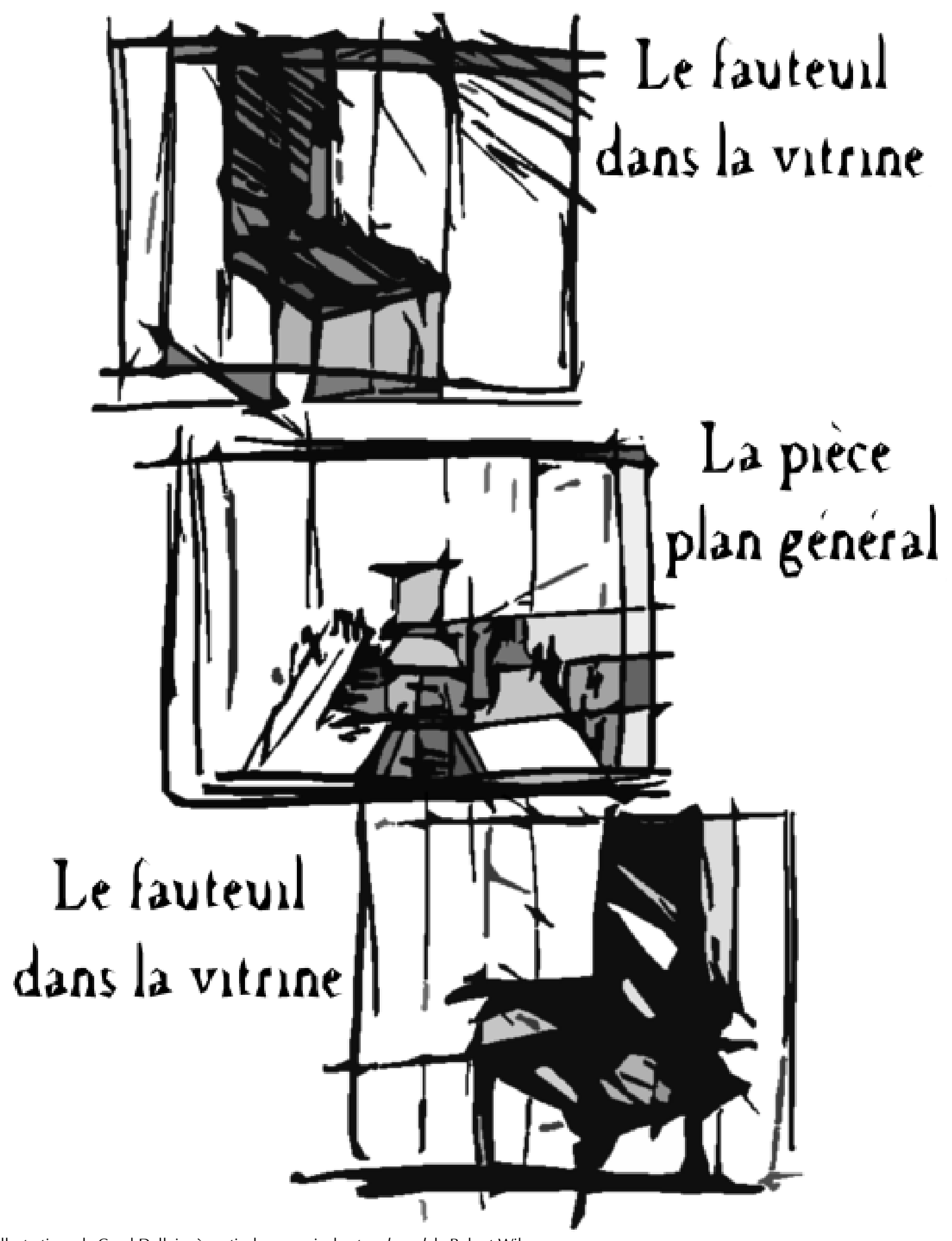

Illustrations de Carol Dallaire à partir des croquis du story board de Robert Wilson. 